\title{
Activation and enhancement of Fredericamycin A production in deepsea-derived Streptomyces somaliensis SCSIO ZH66 by using ribosome engineering and response surface methodology
}

Yonghe Zhang ${ }^{1 \dagger}$, Huiming Huang ${ }^{1+}$, Shanshan $\mathrm{Xu}^{1}$, Bo Wang ${ }^{2}$, Jianhua Ju${ }^{2}$, Huarong $\operatorname{Tan}^{3}$ and Wenli $\mathrm{Li}^{\mathrm{i}^{*}}$

\begin{abstract}
Background: Marine microorganisms are an important source of new drug leads. However, the discovery and sustainable production of these compounds are often hampered due to the unavailable expression of cryptic biosynthetic gene clusters or limited titer. Ribosome engineering and response surface methodology (RSM) integrated strategy was developed in this study to activate cryptic gene cluster in the deepsea-derived Streptomyces somaliensis SCSIO ZH66, and subsequently isolation, structural analysis, and the yield enhancement of the activated compound, anticancer drug lead Fredericamycin A (FDM A), were performed.
\end{abstract}

Results: In order to discover novel natural products from marine Streptomyces strains by genome mining strategy, the deepsea-derived S. somaliensis SCSIO ZH66 was subject to ribosome engineering to activate the expression of cryptic gene clusters. A resistant strain ZH66-RIF1 was thereby obtained with $300 \mu \mathrm{g} / \mathrm{mL}$ rifampicin, which accumulated a brown pigment with cytotoxicity on MS plate while absent in the wild type strain. After screening of fermentation conditions, the compound with pigment was purified and identified to be FDM A, indicating that the activation of a cryptic FDM A biosynthetic gene cluster was taken place in strain ZH66-RIF1, and then it was identified to be ascribed to the mutation of R444H in the $\beta$ subunit of RNA polymerase. To further improve the yield efficiently, nine fermentation medium components were examined for their significance on FDM A production by PlackettBurman design and Box-Behnken design. The optimum medium composition was achieved by RSM strategy, under which the titer of FDM A reached $679.5 \pm 15.8 \mathrm{mg} / \mathrm{L}$ after 7 days of fermentation, representing a 3-fold increase compared to the original medium. In terms of short fermentation time and low-cost fermentation medium, strain ZH66-RIF1 would be an ideal alternative source for FDM A production.

Conclusions: Our results would hasten the efforts for further development of FDM A as a drug candidate. Moreover, this ribosome engineering and RSM integrated methodology is effective, fast and efficient; it would be applicable to genome mining for novel natural products from other strains.

Keywords: Deepsea-derived Streptomyces, Ribosome engineering, Cryptic Gene cluster, Response Surface Methodology (RSM), Fredericamycin A (FDM A)

\footnotetext{
* Correspondence: liwenli@ouc.edu.cn

${ }^{\dagger}$ Equal contributors

"Key Laboratory of Marine Drugs, Ministry of Education of China, School of Medicine and Pharmacy, Ocean University of China, Qingdao 266003, China Full list of author information is available at the end of the article
} 


\section{Introduction}

After years of screening from soil dwelling microorganisms, it is more and more difficult to discover novel compounds through traditional screening strategies, which increases the drug development costs and drains the pipeline of natural product drugs. Marine microorganisms, especially Streptomyces, have become an important source of pharmacologically active metabolites [1,2]. Because marine environmental conditions are extremely different from the terrestrial environment [3], it is surmised that marine microorganisms have different characteristics from their terrestrial counterparts and, therefore, might produce different types of bioactive compounds. Marine Streptomyces are widely distributed in the oceans and, more importantly, novel compounds with biological activities have been isolated from them at a high frequency, indicating that marine Streptomyces are an important source for drug discovery. However, the discovery and sustainable production of bioactive compounds are often hampered due to the unavailable expression of cryptic biosynthetic gene clusters or limited incubation and testing conditions [4].

Over the past decade, actinomycete genome sequencing has revealed that many secondary metabolites biosynthetic gene clusters are "silent" under ordinary laboratory conditions $[5,6]$, which greatly precluded discovery of novel bioactive compounds. Ribosome engineering targeting ribosomal proteins or RNA polymerase (RNAP) has been proved to be effective for waking up and upregulation of the expression of cryptic gene clusters [6-8], leading to discovery of cyclic peptide Piperidamycin A [9] and chlorinated alkaloids Inducamides A-C [10]. In our efforts to discover novel natural products from marine Streptomyces strains by using genome mining strategy, deepsea-derived Streptomyces somaliensis SCSIO ZH66 was isolated, identified and subject to ribosome engineering to activate the cryptic gene clusters in the genome. A rifampicin-resistant mutant strain accumulating dark brown pigment was obtained; the pigment was purified and identified as anticancer drug lead Fredericamycin (FDM) A.

FDM A, first isolated from terrestrial Streptomyces griseus ATCC49344 in 1981 [11], is an aromatic pentadecaketide featuring two sets of peri-hydroxy tricyclic aromatic moieties connected through a unique asymmetric carbaspiro center, representing a new chemotype for anticancer drug leads [12]. The cytotoxicity of FDM A against tumor cell lines resulted from inhibition of DNA topoisomerases I and II as well as the peptidyl-prolyl cis/trans isomerase Pin1, which is involved in the regulation of cell cycle and cell division [13,14]. Given its promising bioactivity and unique structural architecture, many efforts have been devoted to chemical syntheses [15-17] and biosynthesis [18-21] of FDM A. However, the limited yield of FDM A is still one of the major issues precluding its further development as an anticancer drug. Hence, the rifampicin resistant mutant strain ZH66-RIF1 may serve as an alternative efficient FDM A producer. To further improve FDM A production rapidly, response surface methodology (RSM) [22,23] was adopted. Unlike the conventional one-factor-at-a-time method, RSM is a mathematical and statistical method used to describe and predict the response of a multi-variable system with fewer experiments; therefore, it is much more efficient in terms of time and cost [24].

Here we report the ribosome engineering of $S$. somaliensis SCSIO ZH66 resulting in accumulation of dark brown pigment, the identification of the compound with pigment and its encoding gene cluster, and the rapid enhancement of FDM A production using RSM methodology.

\section{Results}

Ribosome engineering of a deepsea-derived Streptomyces strain

S. somaliensis SCSIO ZH66 (CGMCC NO.9492) was isolated from the deep-sea sediment collected at a depth of $3536 \mathrm{~m}$ of the South China Sea. A 1520-bp 16S rRNA gene sequence of this strain was determined and compared against the EzTaxon-e server Database [25]. The result showed that it is highly similar to Streptomyces $16 \mathrm{~S}$ rRNA with 99\% identity to that of Streptomyces somaliensis (GenBank accession number AB184243). Therefore, this strain was named as $S$. somaliensis SCSIO ZH66. Phylogenetic analysis of strain ZH66 with its related Streptomyces type strains is shown in Additional file 1: Figure S1. Considering its unique ecological environment, the genome of strain ZH66 was sequenced, which revealed that at least 20 secondary metabolites biosynthetic gene clusters are existed in this strain (data not shown). To activate the expression of cryptic gene clusters, strain ZH66 was subject to ribosome engineering. Hence, mutant strain ZH66-RIF1 was generated as described in the Materials and methods section, which was screened at the presence of $300 \mu \mathrm{g} / \mathrm{mL}$ rifampicin. In contrast to the wild-type strain ZH66-WT, mutant strain ZH66-RIF1 accumulated dark brown pigment on MS plate (Figure 1A); HPLC analysis of their ethyl acetate extracts revealed production of compound $\mathbf{1}$ by strain ZH66-RIF1 but not by the wild-type strain (Figure 1C). The mutation in strain ZH66-RIF1 was then determined to be R444H in the $r p o B$ gene encoding the $\beta$ subunit of RNAP (Figure 1B). In addition, the morphological differentiation of strain ZH66-RIF1 was obviously impaired. As shown in Additional file 1: Figure S2, the wild-type strain ZH66-WT sporulated well on Gauze's No.1 medium when incubated at $30^{\circ} \mathrm{C}$ for $72 \mathrm{~h}$, while no sporulation was observed for strain ZH66-RIF1 at the 

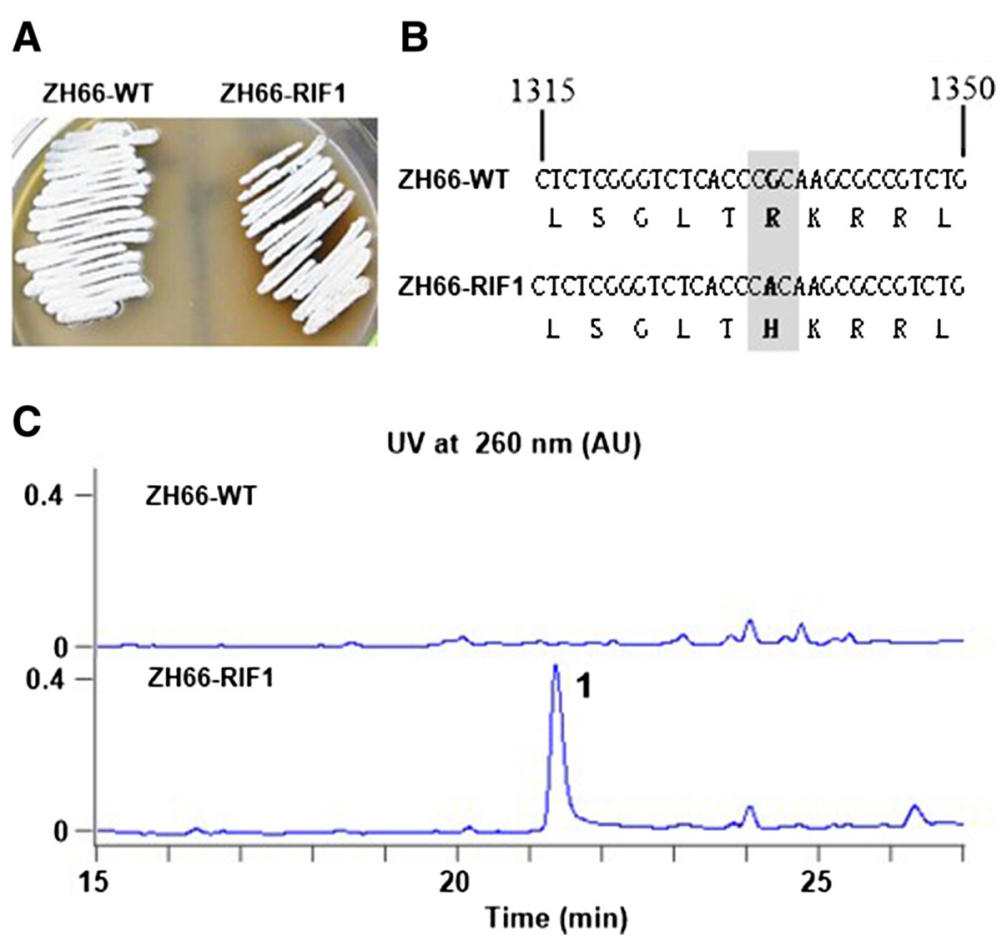

Figure 1 Comparisons of wild-type strain ZH66-WT and rifampicin-resistant mutant strain ZH66-RIF1. (A) Phenotype of strain ZH66-WT and strain ZH66-RIF1 on MS plate when incubated at $30^{\circ} \mathrm{C}$ for 7 days. (B) Comparison of the rpoB genes from strain ZH66-WT and strain ZH66-RIF1. (C) HPLC traces of strain ZH66-WT and strain ZH66-RIF1. "1" indicates the new compound accumulated by strain ZH66-RIF1.

same conditions. Furthermore, the extracts were tested for cytotoxicity against three human cancer cell lines. To our delight, strong inhibitions were observed for strain ZH66-RIF1 with half-effective concentration $\left(\mathrm{EC}_{50}\right)$ of $3.4 \mathrm{mg} / \mathrm{mL}$ against A-549, $1.6 \mathrm{mg} / \mathrm{mL}$ against HCT116, and $3.9 \mathrm{mg} / \mathrm{mL}$ against P388, indicating compound $\mathbf{1}$ probably has significant anticancer bioactivity.

\section{Identification of FDM A production from the rifampicin resistant strain ZH66-RIF1}

Given the important role of medium composition for gene expression [26], strain ZH66-RIF1 was fermented in different medium to compare the production of compound 1 (Additional file 1: Table S1); among them, medium-3 gave rise to the best yield of approximate $220.9 \mathrm{mg} / \mathrm{L}$, which was about 30-, 5-, 10- and 13-fold higher than medium-1 ( 7.2 mg/L), 2 ( 42.1 mg/L), 4 ( 20.9 $\mathrm{mg} / \mathrm{L})$ and $5(\sim 15.6 \mathrm{mg} / \mathrm{L})$, respectively (Additional file 1: Figure S3). Therefore, fermentation (2 L) was carried out using medium-3, from which compound $\mathbf{1}$ was purified and then subject to structural analysis. The UV spectrum of compound 1 displayed $\lambda_{\max }$ at 260, 302, 316, 332, 357, 373 and $392 \mathrm{~nm}$ (Additional file 1: Figure S4), and the chemical formula of compound 1 was determined to be $\mathrm{C}_{30} \mathrm{H}_{21} \mathrm{NO}_{9}$ by HR-ESI-MS (m/z 540.1280 $[\mathrm{M}+\mathrm{H}]^{+}$, calcd 540.1295) (Figure 2B). Both are consistent with those of FDM A [12]. The ${ }^{1} \mathrm{H}-\mathrm{NMR}$ (Figure 2C) and ${ }^{13} \mathrm{C}$-NMR (Figure 2D) data of compound $\mathbf{1}$ were further recorded, and the chemical shifts were summarized in Additional file 1: Table S2. Taken all the above data together, the identity of compound $\mathbf{1}$ was unambiguously confirmed to be the anticancer drug lead FDM A (Figure 2A). Compared with the previously reported FDM A producing strains, FDM A titers from Streptomyces chattanoogensis ISP 5002 [27] and S. griseus ATCC49344 [20] are $10 \mathrm{mg} / \mathrm{L}$ and $162 \mathrm{mg} / \mathrm{L}$, respectively. Therefore, the strain ZH66-RIF1 may serve as an alternative producer with the FDM A titer reaching $220.9 \mathrm{mg} / \mathrm{L}$ when cultured in medium-3 (Additional file 1: Table S3).

\section{Identification of the FDM A biosynthetic gene cluster from strain $\mathrm{ZH} 66$}

In consistent with the production of FDM A, genome analysis of strain $\mathrm{ZH} 66$ revealed the presence of a FDM gene cluster (herein named frd), which covers about 25kb DNA region containing 27 open reading frames (orfs) with remarkably high homology to their homologs from S. griseus ATCC49344 (fdm gene cluster) [18] and Streptomyces sp. SANK61196 (san gene cluster) [28] (Additional file 1: Table S4). As shown in Figure 3, the gene composition and organization of these three clusters are highly conserved but with the following exceptions: (i) $f d m X$ only exists in the $f d m$ gene cluster, which putatively encodes a transposase and probably related 
A
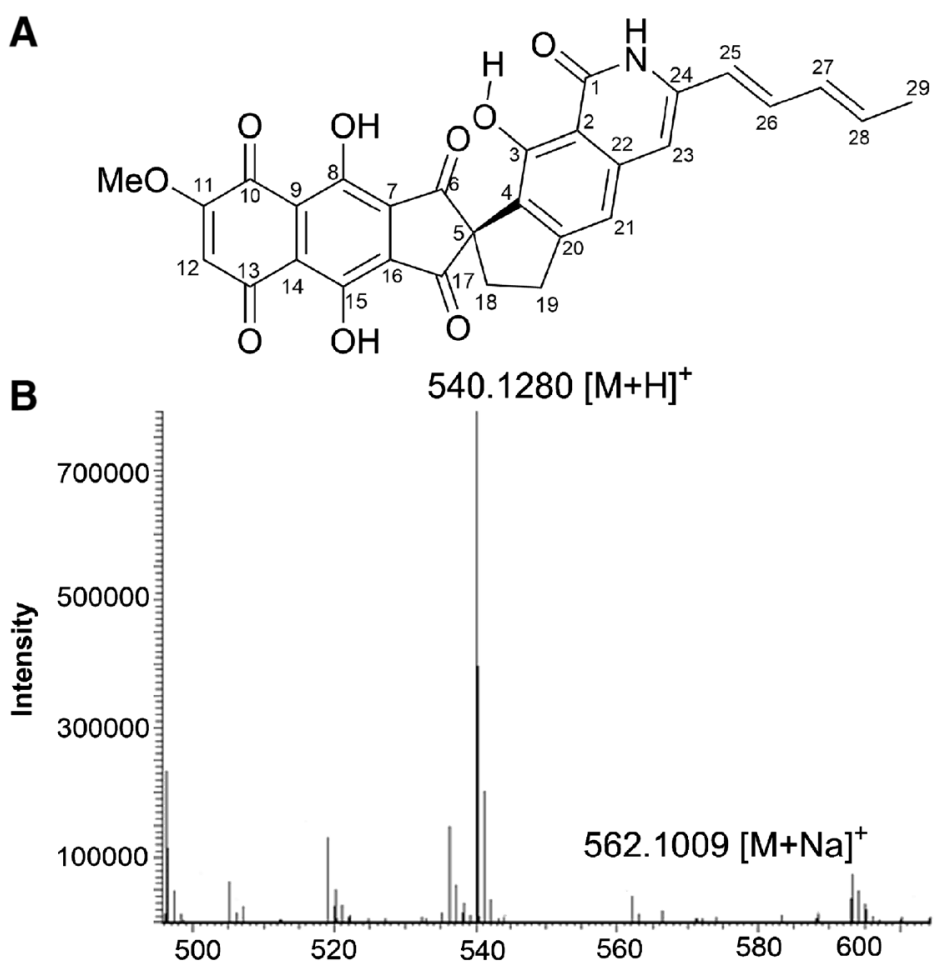

C

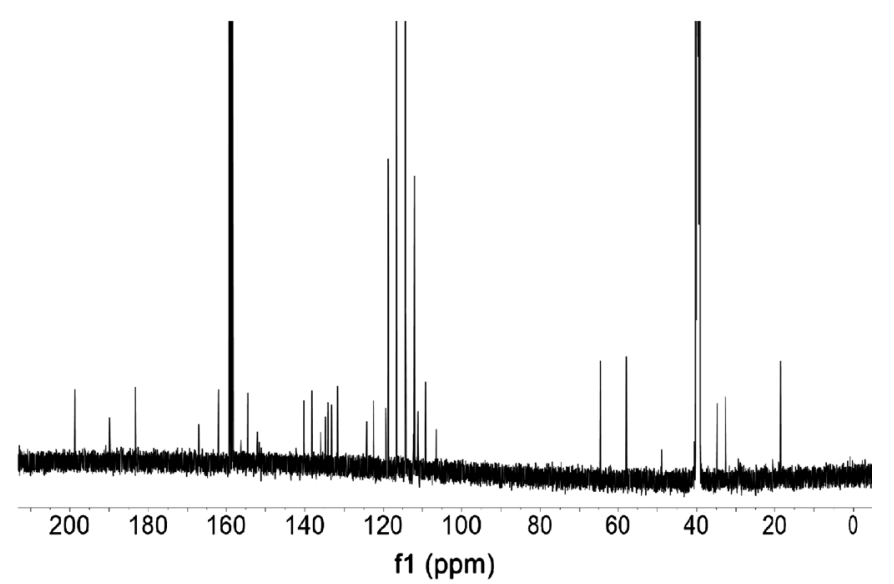

D

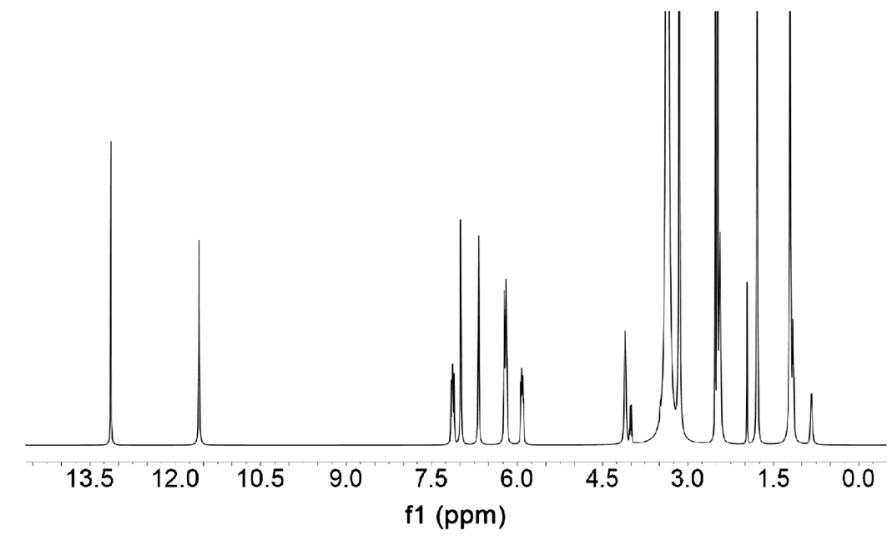

Figure 2 Structure determination of FDM A. (A) Chemical structure of FDM A. (B) HRMS data of FDM A. (C) ${ }^{1} H$ NMR spectrum of FDM A in DMSO- $d_{6}$. (D) ${ }^{13} \mathrm{C}$ NMR spectrum of FDM A in DMSO- $d_{6}$. 


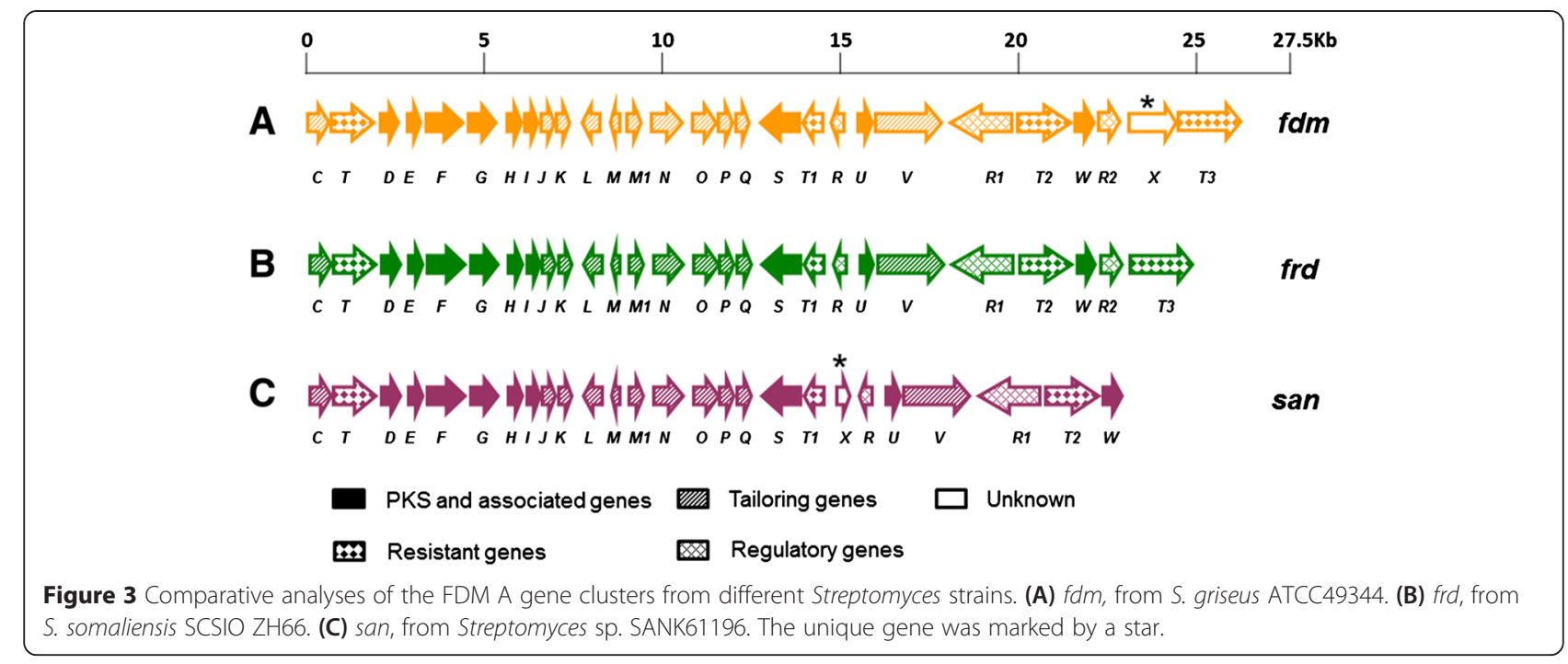

to the horizontal transfer of the gene cluster; (ii) $\operatorname{san} X$ with unknown function is unique for the san gene cluster; (iii) the counterparts of $f d m R 2 / f r d R 2$ and $f d m T 3 /$ frdT3 are absent in the san gene cluster. The sequence was deposited in the GenBank database under the accession number KP213175. The production of FDM A in strain ZH66-RIF1 but not in strain ZH66 suggested activation of the cryptic frd gene cluster. The transcription levels of the frdD gene (encoding polyketide cyclase) and the frdR1 gene (encoding Streptomyces antibiotic regulatory protein, SARP) (Additional file 1: Table S4, S5) were further analyzed by RT-PCR (Figure 4A) and real time RT-PCR (Figure 4B), confirming that both of them were substantially transcribed in strain ZH66-RIF1 in comparison with the wild-type strain ZH66.
Screening of significant variables for FDM A production using Plackett-Burman design (PBD)

To further improve FDM A production efficiently, we screened the significant variables for FDM A production using PBD. As shown in Additional file 1: Table S6, nine different factors were tested, including soluble starch $\left(X_{1}\right)$, glucose $\left(X_{2}\right)$, corn syrup $\left(X_{3}\right)$, yeast extract $\left(X_{4}\right)$, beef extract $\left(X_{5}\right), \mathrm{CaCO}_{3}\left(X_{6}\right), \mathrm{MgSO}_{4} \cdot 7 \mathrm{H}_{2} \mathrm{O}\left(X_{7}\right)$, $\mathrm{KH}_{2} \mathrm{PO}_{4}\left(X_{8}\right)$ and sea salt $\left(X_{9}\right)$. All the experiments were performed in triplicate and the average of results (FDM A titer) were presented as the response in Additional file 1: Table S6. The effect on FDM A production was estimated for each variable (Additional file 1: Table S7). The Pareto chart (Figure 5) clearly showed that soluble starch $\left(X_{1}\right)$, glucose $\left(X_{2}\right)$, and $\mathrm{KH}_{2} \mathrm{PO}_{4}\left(X_{8}\right)$ had confidence
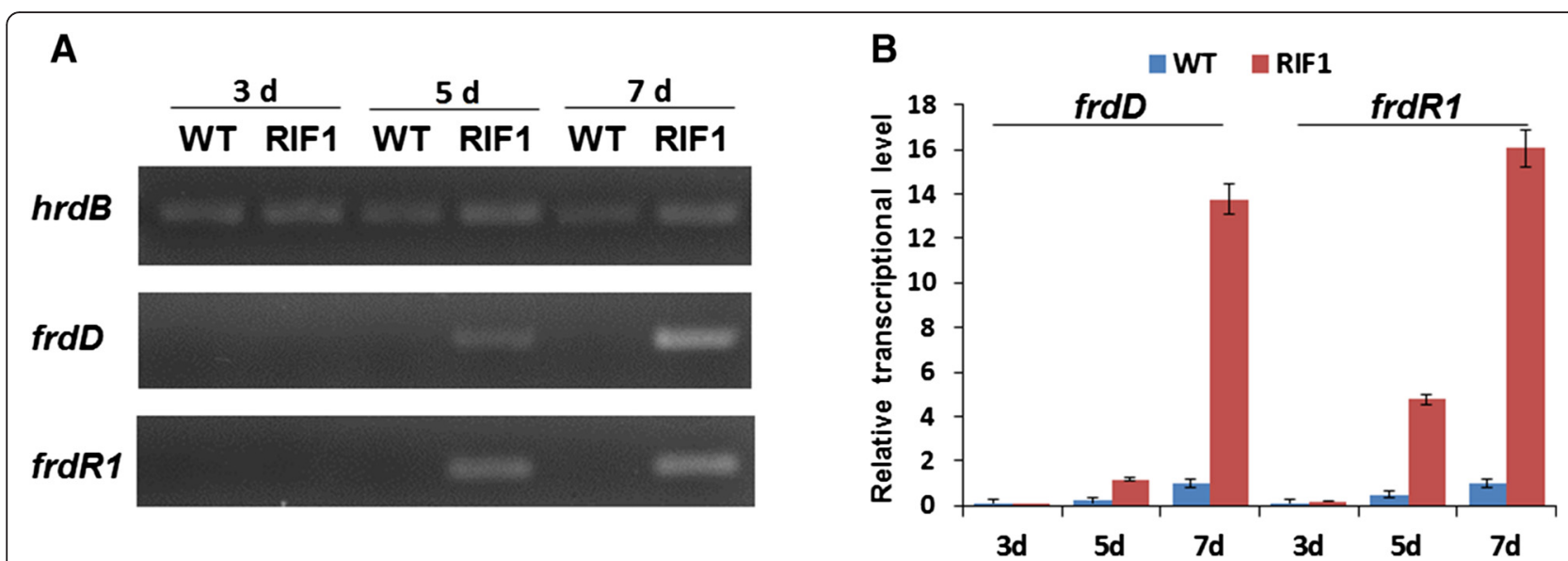

Figure 4 Transcriptional analysis of frdD and frdR1 genes in wild-type strain ZH66-WT and rifampicin-resistant mutant strain ZH66-RIF1. Transcripts were determined by RT-PCR (A) and real-time RT-PCR (B). The transcription levels were detected at 3,5 and 7 days in strain ZH66-WT and strain ZH66-RIF1 grown on MS plates. Error bars indicated standard deviations $(n=3)$. 


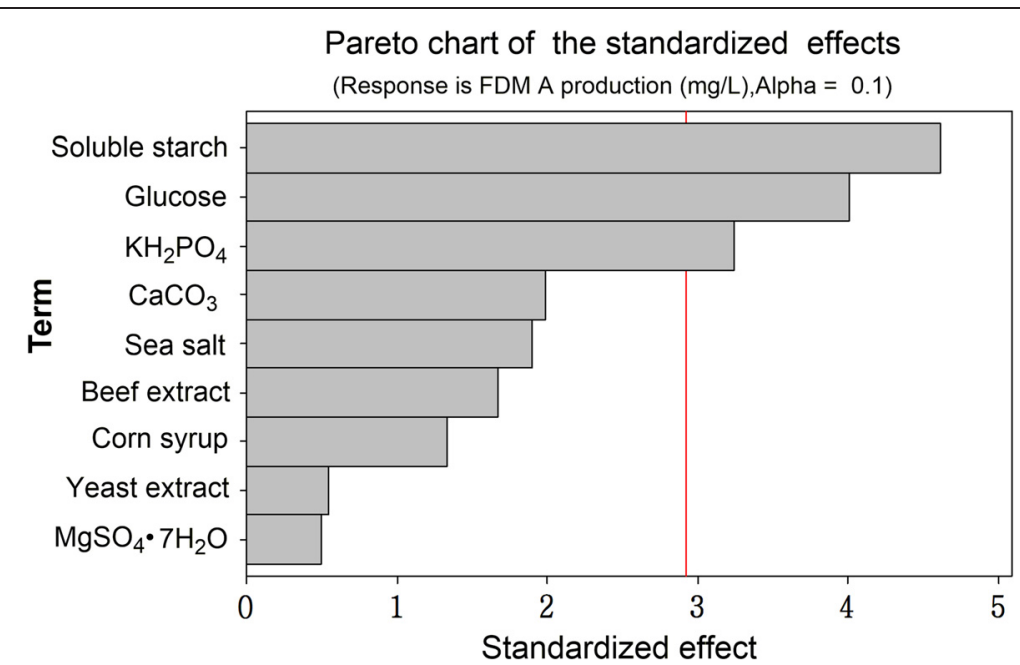

Figure 5 The effects of different factors on FDM A production. The bars of the diagram which go beyond the vertical red line correspond to the statistically significant effects, for a confidence level of $90 \%$.

levels above $90 \%(p<0.01)$. Therefore, these three variables were selected for further optimization.

\section{Optimization of FDM A production using response surface methodology}

To determine the optimal levels of the above selected variables, the RSM based on Box-Behnken design (BBD) was employed. The respective coded levels for each factor were listed in Additional file 1: Table S8. The experimental design and the corresponding results were shown in Table 1. By employing multiple regression analysis on the experimental data, the following equation

Table 1 The design of experiments and response of FDM A production

\begin{tabular}{|c|c|c|c|c|}
\hline Run & $\frac{\text { Soluble starch }}{x_{1}}$ & $\frac{\text { Glucose }}{X_{2}}$ & $\frac{\mathrm{KH}_{2} \mathrm{PO}_{4}}{X_{8}}$ & $\begin{array}{l}\text { FDM A titer } \\
(Y, \mathrm{mg} / \mathrm{L})\end{array}$ \\
\hline 1 & -1 & -1 & 0 & $531.49 \pm 5.21$ \\
\hline 2 & -1 & 1 & 0 & $675.39 \pm 20.00$ \\
\hline 3 & 0 & 1 & -1 & $684.50 \pm 8.08$ \\
\hline 4 & 1 & -1 & 0 & $334.49 \pm 19.05$ \\
\hline 5 & 0 & 1 & 1 & $548.14 \pm 9.34$ \\
\hline 6 & 0 & 0 & 0 & $633.26 \pm 17.38$ \\
\hline 7 & 0 & 0 & 0 & $637.19 \pm 8.79$ \\
\hline 8 & 1 & 1 & 0 & $484.39 \pm 3.57$ \\
\hline 9 & 0 & -1 & -1 & $513.95 \pm 6.54$ \\
\hline 10 & 1 & 0 & 1 & $364.40 \pm 4.06$ \\
\hline 11 & 0 & -1 & 1 & $398.01 \pm 7.40$ \\
\hline 12 & 1 & 0 & -1 & $533.37 \pm 3.95$ \\
\hline 13 & 0 & 0 & 0 & $628.13 \pm 10.49$ \\
\hline 14 & -1 & 0 & 1 & $578.48 \pm 8.97$ \\
\hline 15 & -1 & 0 & -1 & $647.68 \pm 8.08$ \\
\hline
\end{tabular}

was obtained, describing the relationship between FDM A titer $(Y)$ and the tested variables $(X)$ :

$$
\begin{aligned}
Y= & 636.19-84.80 X_{1}+76.81 X_{2}-66.06 X_{8}-65.21 X_{1}{ }^{2}-64,543 X_{2}{ }^{2} \\
& -35.5 X_{8}{ }^{2}-29.44 X_{1} X_{8}+1.5 X_{1} X_{2}-5.105 X_{2} X_{8}
\end{aligned}
$$

Where $Y$ was the predicted FDM A titer; $X_{1}, X_{2}$ and $X_{8}$ were the concentrations of soluble starch, glucose and $\mathrm{KH}_{2} \mathrm{PO}_{4}$, respectively.

To validate the regression coefficient, analysis of variance (ANOVA) for FDM A production was performed. The high $F$-value $(62.72)$ and low $p$-value $(0.000<0.05)$ implied the model was highly significant (Additional file 1: Table S9). As shown in Table 2, most regression coefficients were highly significant with $p$-values less than 0.05 . The regression analysis of the data showed coefficient of determination $\left(\mathrm{R}^{2}\right)$ value of 0.9912 and adjusted $R^{2}$ value of 0.9754 , which were in close agreement.

Table 2 The least-squares fit and coefficient estimate

\begin{tabular}{lllll}
\hline Variables & Coefficient estimate & Standard error & $\boldsymbol{t}$-value & $\boldsymbol{p}$-value \\
\hline Intercept & 636.193 & 10.156 & 62.644 & 0.000 \\
$X_{1}$ & -84.799 & 6.219 & -13.635 & 0.000 \\
$X_{2}$ & 76.810 & 6.219 & 12.351 & 0.000 \\
$X_{8}$ & -66.059 & 6.219 & -10.622 & 0.000 \\
$X_{1}{ }^{2}$ & -65.210 & 9.154 & -7.124 & 0.001 \\
$X_{2}^{2}$ & -64.543 & 9.154 & -7.051 & 0.001 \\
$X_{8}{ }^{2}$ & -35.500 & 9.154 & -3.878 & 0.012 \\
$X_{1} X_{2}$ & 1.500 & 8.795 & 0.171 & 0.871 \\
$X_{1} X_{8}$ & -29.442 & 8.795 & -3.348 & 0.020 \\
$X_{2} X_{8}$ & -5.105 & 8.795 & -0.580 & 0.587 \\
\hline $\mathrm{R}^{2}=99.12 \% ; \mathrm{R}^{2} \mathrm{adj}=97.54 \%$. & & &
\end{tabular}


Therefore, the present $R^{2}$ value reflected a very good fit between the observed and predicted responses, indicating that the model was reliable for FDM A production in the present study.

The 3D response surfaces and 2D contour plots (Figure 6) were the graphical representations of the regression equation, which indicated the optimum ranges of the variables for the maximal response. Clearly, the FMD A production increased with increase of glucose concentration (level $-1 \sim 0.6$ ), and decrease of soluble starch (level $1 \sim-0.75$ ) and $\mathrm{KH}_{2} \mathrm{PO}_{4}$ (level $1 \sim-0.7$ ) concentrations.

\section{Validation of the optimized condition}

Based on the above optimized medium composition, the maximum yield of FDM A was predicted to be $699.84 \mathrm{mg} / \mathrm{L}$ when the code levels of $X_{1}, X_{2}$ and $X_{8}$ were -0.724 (soluble starch $5.55 \mathrm{~g} / \mathrm{L}$ ), 0.446 (glucose $27.78 \mathrm{~g} / \mathrm{L})$, and $-0.706\left(\mathrm{KH}_{2} \mathrm{PO}_{4} 0.301 \mathrm{~g} / \mathrm{L}\right)$, respectively. To confirm the predicted results, validation experiments
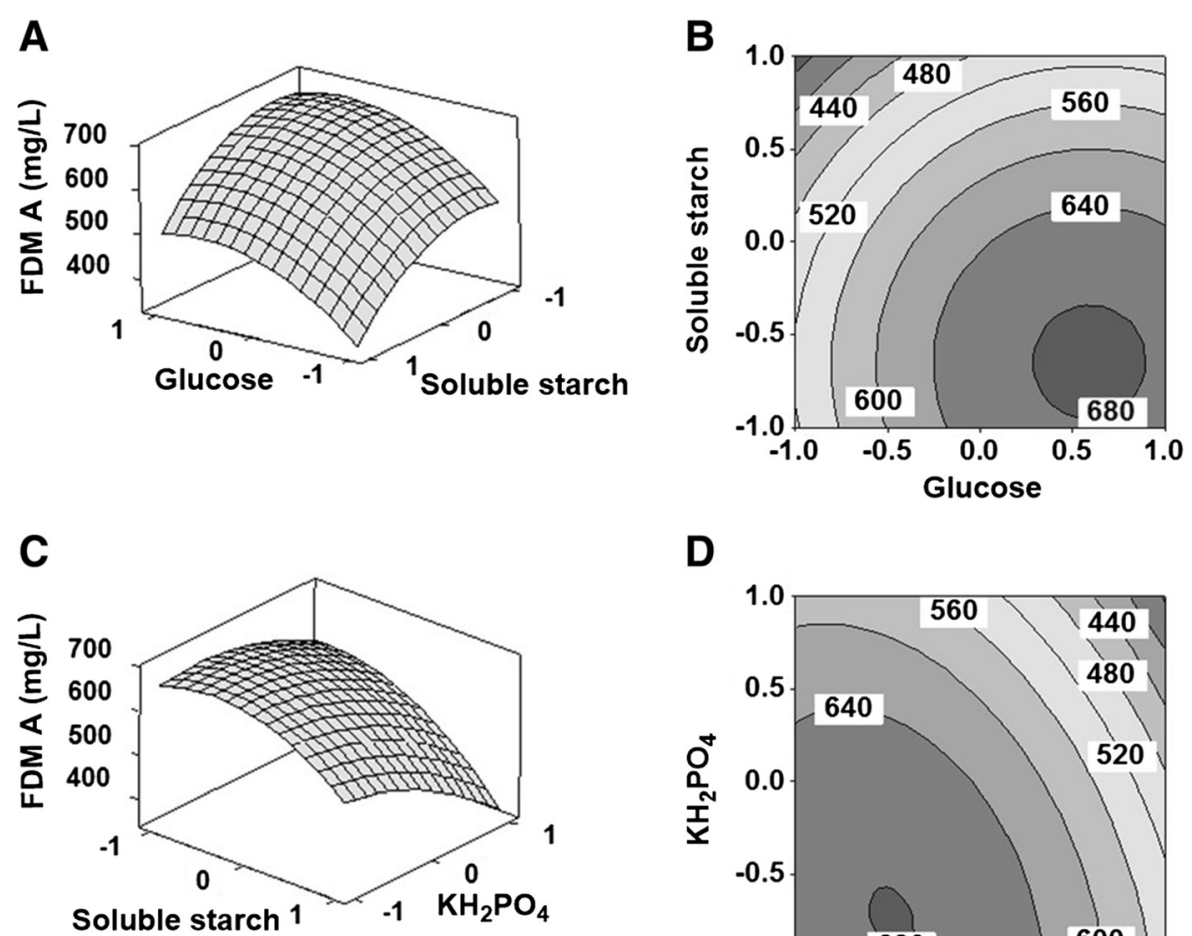

D
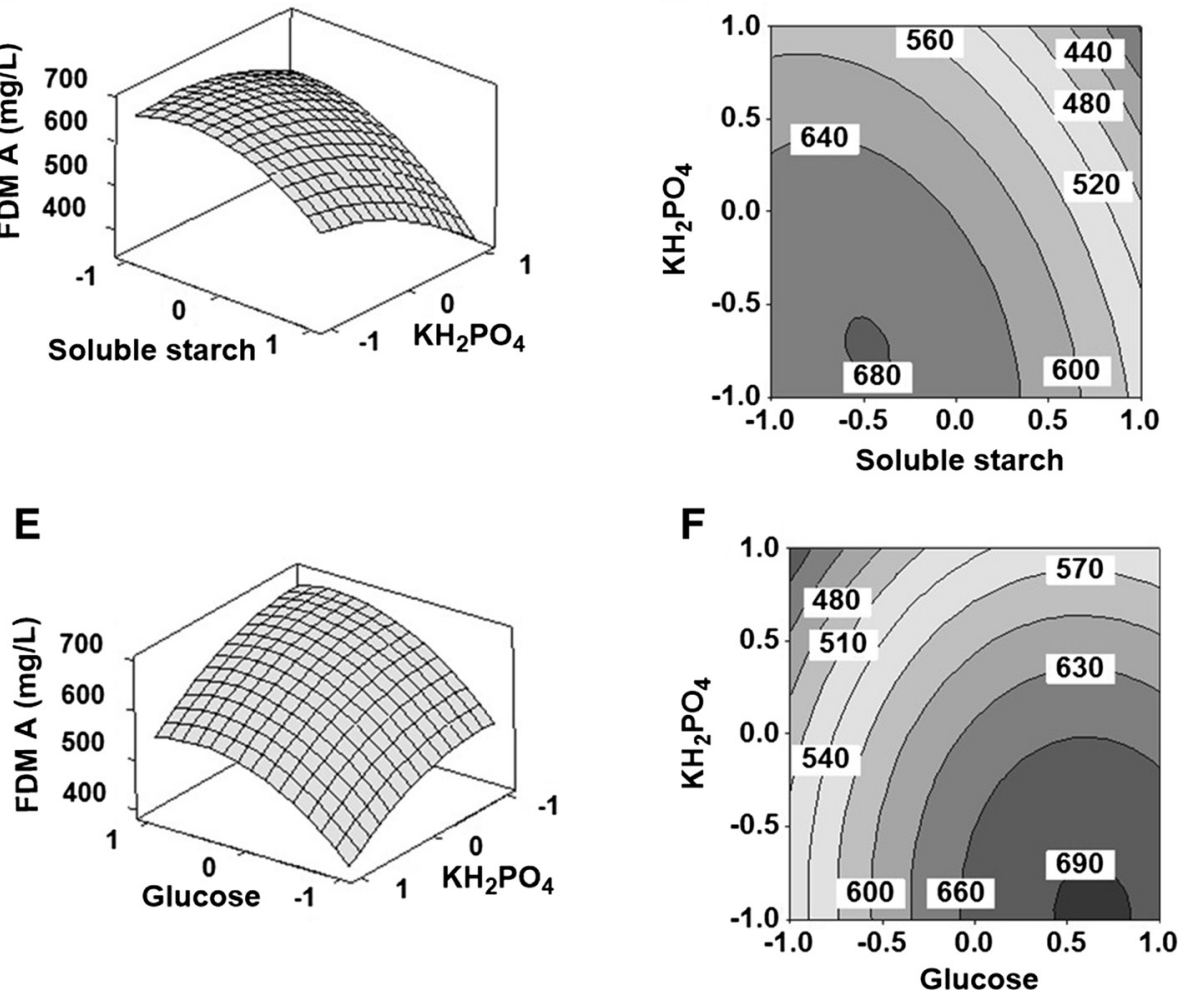

Figure $63 \mathrm{D}$ response surfaces and 2D contour plots for FDM A production. Each figure presented the effect of two variables on the production of FDM A, while the other one variable was held at zero level. $(\mathbf{A}, \mathbf{B})$ The effects of soluble starch $\left(X_{1}\right)$ and glucose $\left(X_{2}\right)$ on FDM A production. $(\mathbf{C}, \mathbf{D})$ The effects of soluble starch $\left(X_{1}\right)$ and $\mathrm{KH}_{2} \mathrm{PO}_{4}\left(X_{8}\right)$ on FDM A production. (E, F) The effects of glucose $\left(X_{2}\right)$ and $\mathrm{KH}_{2} \mathrm{PO}_{4}\left(X_{8}\right)$ on FDM A production. 
were performed in triplicate. Under the optimal conditions, the observed experimental average concentration of FDM A was $679.5 \pm 15.82 \mathrm{mg} / \mathrm{L}$, suggesting that the experimental and the predicted values $(699.84 \mathrm{mg} / \mathrm{L})$ were in good agreement.

\section{Discussion}

Activation of cryptic secondary metabolite biosynthetic gene clusters and improvement of production of potential drug leads are two important objects for drug discovery and development [6,7]. Ribosome engineering is an effective strategy for waking up dormant gene clusters, but the titer of the activated compounds may still be limited for further chemical and biological evaluations, which would severely hamper their development as drug candidates. In the present study, a Streptomyces strain was isolated from the deep sea, and was identified as S. somaliensis SCSIO ZH66 based on the $16 \mathrm{~S}$ rDNA sequence (Additional file 1: Figure S1). A ribosome engineering and RSM integrated strategy was developed to activate cryptic gene clusters in strain ZH66, and subsequently enhance the titer of the activated anticancer drug lead FDM A (Figure 2), reaching a considerable yield of $679.5 \pm 15.8 \mathrm{mg} / \mathrm{L}$ after 7 days of fermentation.

Ribosome engineering is performed using antibiotics that target either the ribosome itself or RNAP. Drugresistant mutants enforced by rifampicin harbor mutations in $r p o B$ gene (encoding the $\beta$ subunit of RNAP) $[6,7]$. Recently, Fu et al. reported the discovery of chlorinated alkaloids Inducamides $\mathrm{A}-\mathrm{C}$ from the rifampicinresistant strain Streptomyces sp. SNC-109-M3, which was identified to contain a S442F mutation in rpoB gene [10]; while mutant strain ZH66-RIF1 contained a mutation of $\mathrm{R} 444 \mathrm{H}$, which was probably responsible for activating FDM A production (Figures 1 and 4) and reducing the growth rate of strain ZH66RIF1 as well (Additional file 1: Figure S2). Notwithstanding, additional mutations might also exist in the genome of strain ZH66-RIF1 besides the mutation in RNAP. Notably, activation of cryptic gene clusters by rроB mutations was mediumdependent [9], indicating the important roles of medium composition for the expression of the activated gene clusters. Hence, strain ZH66-RIF1 was cultured under different conditions, and the yield of FDM A varied significantly in various medium (Additional file 1: Figure S3). We assume other compounds with novel structures might also produce if more culture conditions are screened. In addition, experimental tools such as HPLC-MS/MS, RT-PCR would probably be necessary to access the full profile of the activated gene clusters in strain ZH66-RIF1.

Although strain ZH66 was isolated from the deep sea, the frd gene cluster exhibited surprisingly high homology to those from S. griseus ATCC49344 and Streptomyces sp. SANK61196 (Figure 3, Additional file 1: Table S1). The presence of $f d m X$ gene, presumably encoding a transposase, indicated that the $f d m$ gene cluster in $S$. griseus ATCC49344 was probably obtained via horizontal gene transfer (HGT) events from other strains. Since the homology of frd vs $f d m$ (98-100\% identity/similarity on amino acids level) is much higher than that of frd vs san (62-94\% identity/similarity on amino acids level), the frd gene cluster should have closer evolution relationship with the $f d m$ gene cluster than with the san gene cluster. Intriguingly, in addition to FDM A, the san gene cluster was reported to encode a structurally distinct compound A-74528 as well [29]. Nevertheless, strain ZH66 represented the first FDM A producer isolated from marine environment. Given its promising value as anticancer drug candidate, enhancement of the FDM A titer was of interest for its further development. RSM strategy was adopted to evaluate multiple variables efficiently, leading to establishment of the optimized medium composition for FDM A production (Table 1, Figure 6). Notably, compared with the original medium, the ratio of soluble starch (slow-acting) to glucose (fastacting) was changed from $1: 2(10 \mathrm{~g} / \mathrm{L}$ to $20 \mathrm{~g} / \mathrm{L})$ to approximately $1: 5(5.55 \mathrm{~g} / \mathrm{L}$ to $27.78 \mathrm{~g} / \mathrm{L})$, suggesting that low concentration of glucose probably could not meet the requirement of cell growth thus leading to low yield of FDM A.

By overexpressing the SARP-type activator gene $f d m R 1$ in S. griseus ATCC49344, the titer of FDM A reached about $997 \mathrm{mg} / \mathrm{L}$ when cultured in APM medium (medium-2, Additional file 1: Table S1) for 10 days; since a two-stage fermentation process was adopted, the total fermentation took 11 to 12 days plus 1 or 2 days for seed culture; conversely, the titer of FDM A was about only $400 \mathrm{mg} / \mathrm{L}$ on day 7 (not including seed culture) (Additional file 1: Table S3) [20]. In the present study, when cultured in APM medium, the FDM A titer was only one fifth of that in medium-3 (Additional file 1: Figure S3); a titer of $679.5 \pm 15.82 \mathrm{mg} / \mathrm{L}$ was achieved in the optimized medium on day 7 in a one-stage fermentation process. In terms of the medium composition, our optimized medium is much cheaper than APM medium. Therefore, taking into account of the medium cost and fermentation time, strain ZH66-RIF1 would be an ideal FDM A producer, which could be expedient to its pharmaceutical development.

\section{Conclusions}

Marine Streptomyces strains serve as important sources for novel secondary metabolites. However, many secondary metabolites biosynthetic gene clusters are "silent" in typical lab conditions. Ribosome engineering was performed to wake up the cryptic gene clusters in the deepsea-derived S. somaliensis SCSIO ZH66, leading to 
activation of a FDM biosynthetic gene cluster, which showed high homology to the ones from $S$. griseus ATCC49344 and Streptomyces sp. SANK61196. RSM strategy was further adopted to rapidly improve FDM A accumulation. Among the variables, soluble starch, glucose and $\mathrm{KH}_{2} \mathrm{PO}_{4}$ were found to be the most significant. A maximum FDM A production of $679.5 \pm$ $15.82 \mathrm{mg} / \mathrm{L}$ was achieved with the optimized medium developed by RSM, as compared to $220.9 \mathrm{mg} / \mathrm{L}$ in the original medium. Since FDM A was a potential anticancer drug, the present study provided a new FDM A producing marine Streptomyces stain and a more feasible and inexpensive medium for further large scale fermentation. More importantly, ribosome engineering and RSM integrated methodology is effective, fast and efficient for activation of cryptic gene cluster and the subsequent titer optimization, which would facilitate novel compounds discovery and their sufficient production for further pharmaceutical development.

\section{Material and methods}

Microorganism and culture conditions

S. somaliensis SCSIO ZH66 (CGMCC NO.9492) was isolated from the deep sea sediment collected at a depth of 3536 meters of the South China Sea $\left(120^{\circ} 0.250^{\prime} \mathrm{E} ; 20^{\circ}\right.$ $\left.22.971^{\prime} \mathrm{N}\right)$. The strain was grown at $30^{\circ} \mathrm{C}$ on $\mathrm{MS}$ medium for sporulation. Five fermentation media as described in the supplemental materials (Additional file 1: Table S1) were screened to detect the FDM A production, among which medium-2 was chosen as the start medium for further optimization. For each fermentation, strain ZH66RIF-1 spores were inoculated into $50 \mathrm{~mL}$ of fermentation medium supplemented with $2 \%$ XAD-16 resin in a $250 \mathrm{~mL}$ flask, and incubated at $30^{\circ} \mathrm{C}, 240 \mathrm{rpm}$ for 7 days.

\section{Identification of the actinomycete strain}

Genomic DNA was prepared according to the literature protocol [29]. The 1520-bp 16S rRNA gene was amplified by PCR using the primer pair of $16 \mathrm{~S}-\mathrm{FP} / 16 \mathrm{~S}-\mathrm{RP}$ (Additional file 1: Table S4). The 16S rRNA gene sequence was compared against the EzTaxon-e server Database [25] to retrieve the most similar sequences of type strains. Phylogenetic analysis of the 16S rRNA gene sequences were performed using the Molecular Evolutionary Genetics Analysis (MEGA) software [30], and the substitution model was Poisson correction. Primer synthesis and DNA sequencing were performed at Sunny Biotech Co. Ltd. (Shanghai, China).

\section{Ribosome engineering of strain $\mathrm{ZH} 66$}

Firstly, the minimum inhibitory concentrations (MIC) of rifampicin against strain ZH66 were determined. Spore suspensions $\left(10^{6}\right.$ spores $)$ were spread onto MS plates containing different concentrations $(0-50 \mu \mathrm{g} / \mathrm{mL})$ of rifampicin. The plates were incubated at $30^{\circ} \mathrm{C}$ for 5 days. The minimum concentration that fully inhibited the growth of strain ZH66 was defined as the MIC value, which turned out to be $10 \mu \mathrm{g} / \mathrm{mL}$. Spore suspensions $\left(10^{9}\right.$ spores) were then spread onto MS plates containing rifampicin at concentrations of 30 to $300 \mu \mathrm{g} / \mathrm{mL}$. Mutant colonies producing the darkest pigment were selected, generating mutant strain ZH66-RIF1 which was obtained on the MS plate containing $300 \mu \mathrm{g} / \mathrm{mL}$ rifampicin. To map the mutation in the genome of strain ZH66RIF-1, the rpoB gene was amplified using Q5 highfidelity DNA polymerase (New England Biolabs, Beverly, MA, USA) with the primer pair of rpoB-FP/rpoB-RP (Additional file 1: Table S4). The amplified DNA fragment was digested with BamHI and HindIII, and then was ligated into the same sites of pUC18 to yield pUC18::rpoB-RIF1. After confirmation by enzymatic digestion, three clones were subject to sequencing to analyze the mutation in rpoB gene.

\section{Genomic scanning and sequence analysis}

Genomic DNA was shotgun sequenced using commercial 454 technology by Shanghai Majorbio Bio-pharm Technology Co., Ltd. (Shanghai, China). orf assignments and their proposed function were accomplished by using the FramePlot 4.0beta (http://nocardia.nih.go.jp/fp4) [31] and Blast programs (http://blast.ncbi.nlm.nih.gov/Blast. cgi) [32], respectively.

\section{Biological assays}

Cytotoxicity assays were performed at the Molecular Screening Facility at School of Pharmacy, Ocean University of China (Qingdao, China), by using the SRB method (against A-549 and HCT116), or the MTT method (against P388).

\section{Production and analyses of FDM A}

The fermentation cultures were harvested by centrifugation, and the supernatant was extracted twice with an equal volume of ethyl acetate. The combined EtOAc extracts were concentrated in vacuo to afford residue A. The precipitated mycelia and XAD-16 resin were extracted twice with acetone. The extracts were combined, and acetone was evaporated in vacuo to yield residue B. The combined residues were dissolved in 95:5 mixture of $\mathrm{CHCl}_{2}-\mathrm{MeOH}$, filtered through a $0.2 \mu \mathrm{m}$ filter, and subject to HPLC. The HPLC system consisted of Agilent 1260 Infinity Quaternary pumps and a 1260 Infinity diode-array detector. Analytical HPLC was performed on an Eclipse C18 column $(5 \mu \mathrm{m}, 4.6 \times 150 \mathrm{~mm})$ developed with a linear gradient from $65 \%$ to $100 \% \mathrm{~B} / \mathrm{A}$ (phase A: $0.1 \%$ formic acid in $\mathrm{H}_{2} \mathrm{O}$; phase B: $100 \%$ acetonitrile supplemented with $0.1 \%$ formic acid) in $20 \mathrm{~min}$ 
followed by an additional $10 \mathrm{~min}$ at $100 \% \mathrm{~B}$ at flow rate of $1 \mathrm{~mL} / \mathrm{min}$ and UV detection at $260 \mathrm{~nm}$. For FDM A purification, semi-preparative HPLC was carried out using an YMC-Pack ODS-A C18 column $(5 \mu \mathrm{m}, 120 \mathrm{~nm}$, $250 \times 10 \mathrm{~mm}$ ). Samples were eluted with a linear gradient from $50 \%$ to $95 \% \mathrm{~B} / \mathrm{A}$ in $40 \mathrm{~min}$, followed by $100 \% \mathrm{~B}$ for $10 \mathrm{~min}$ at a flow rate of $2.0 \mathrm{~mL} / \mathrm{min}$ and UV detection at $260 \mathrm{~nm}$. The identity of FDM A was confirmed by HRMS and NMR analysis. HRMS was carried out on Thermo LTQ-XL mass spectrometer. NMR data was recorded with a Bruker Avance 600 spectrometer.

\section{Transcriptional analysis by RT-PCR and real-time RT-PCR}

Total RNAs were prepared using Ultrapure RNA Kit (CWBio. Inc., Beijing, China). The genomic DNA was removed with gDNA Eraser (Takara Bio, Inc., Dalian, China). Synthesis of the first strand cDNA was performed with PrimeScript RT reagent Kit (Takara Bio, Inc., Dalian, China). The reaction parameters of reverse transcription PCR (RT-PCR) were as follows: $95^{\circ} \mathrm{C}$ for $5 \mathrm{~min}$, followed by 30 cycles consisting of $95^{\circ} \mathrm{C}$ for $30 \mathrm{~s}$, $60^{\circ} \mathrm{C}$ for $30 \mathrm{~s}, 72^{\circ} \mathrm{C}$ for $45 \mathrm{~s}$ and a final extension of $72^{\circ} \mathrm{C}$ for $10 \mathrm{~min}$. Quantitative real-time reverse transcription PCR (real-time RT-PCR) was performed on an ABI7500 real-time PCR system (Applied Biosystems) in triplicate for each sample using the SYBR green method. Each reaction mixture contained $5 \mu \mathrm{L}$ of SYBR Premix Ex Taq (Takara Bio, Inc., Dalian, China), $0.2 \mu \mathrm{L}$ of ROX Reference Dye II $(50 \times), 0.5 \mu \mathrm{L}$ of 2.5 -fold diluted cDNA, $0.2 \mu \mathrm{L}$ of each primer at a concentration of $0.2 \mathrm{pmol} \mu \mathrm{L}^{-1}$ (Additional file 1: Table S4) and 3.4 $\mu \mathrm{L} \mathrm{dd}_{2} \mathrm{O}$. The PCR procedures were as follows: $95^{\circ} \mathrm{C}$ for $10 \mathrm{~min}, 40$ cycles $95^{\circ} \mathrm{C}$ for $15 \mathrm{~s}$ and $60^{\circ} \mathrm{C}$ for $1 \mathrm{~min}$. The $h r d B$ gene (Additional file 1: Table S4) was used as the reference for RT-PCR and real-time RT-PCR.

\section{Identifying the significant variables using Plackett-Burman design}

Preliminary investigation of factors affecting the FDM A production was done by using PBD [33]. The variables chosen for the present study were investigated at two widely spaced intervals designated as -1 (low level) and +1 (high level). The experimental design for screening the medium components was shown in Additional file 1 : Table S6. The effects of individual parameters on FDM A production were calculated by the following equation:

$$
\mathrm{E}=\left(\sum \mathrm{M}^{+}-\sum \mathrm{M}^{-}\right) / \mathrm{N}
$$

Where, $\mathrm{E}$ is the effect of parameter under study and $\mathrm{M}^{+}$ and $\mathrm{M}^{-}$are responses (FDM A titer) of trials at which the parameter was at its higher and lower levels, respectively, and $\mathrm{N}$ is the total number of trials.

\section{Optimization by Box-Behnken design}

Levels of the significant parameters and the interaction effects between various media constituents, which significantly influence FDM A production, were analyzed and optimized by BBD $[34,35]$. In this study, the experimental plan consisted of 15 trials and the independent variables were studied at three different levels $(-1,0,1)$. All the experiments were done in triplicate and the average of FDM A production obtained was taken as the response $(Y)$. The second order polynomial coefficients were calculated and analyzed using Minitab16. The general form of the second degree polynomial equation is:

$$
Y=\beta_{0}+\sum \beta_{i} X_{i}+\sum \beta_{i i} X^{2}{ }_{i}+\sum \beta_{i j} X_{i} X_{j}
$$

Where, $Y$ is the predicted response, $X_{i} X_{j}$ are input variables which influence the response variable $Y ; \beta_{0}$ is the offset term; $\beta_{i}$ is the ${ }_{i}$ th linear coefficient; $\beta_{i i}$ is the ${ }_{i}$ th quadratic coefficient and $\beta_{i j}$ is the ${ }_{i j}$ th interaction coefficient. Statistical analysis of the model was performed in the form of ANOVA. This analysis included the Fisher's $F$-test (overall model significance), its associated probability $P(F)$, correlation coefficient $\mathrm{R}$, and determination coefficient $R^{2}$ which measures the goodness of fit of regression model. For each variable, the quadratic models were represented as counter plots and response surface curves which were generated using Minitab16.

\section{Software for experimental design}

The software Minitab16 was used for generation and evaluation of the statistical experimental design. The optimal medium composition for FDM A production was obtained by solving the regression equation and by analyzing the response surface contour plots using the same software.

\section{Nucleotide sequence accession number}

The nucleotide sequences of the 16S rRNA gene, the $r p o B$ gene and the frd gene cluster reported in this paper have been deposited in the GenBank database under accession numbers of KB146142, KP722021 and KP213175, respectively.

\section{Additional file}

Additional file 1: Table S1. Fermentation media used for detecting FDM A production by strain ZH66-RIF1. Table S2. ${ }^{1} \mathrm{H}$ and ${ }^{13} \mathrm{C}$ NMR data of FDM A in DMSO- $d_{6}$. Table S3. FDM A titers from different producing strains. Table S4. The frd biosynthetic gene cluster from S. somaliensis SCSIO ZH66. Table S5. The primer pairs used in this study. Table S6. Screening of significant variables for FDM A production using PlackettBurman Design (PBD). Table S7. The effects of each factor on FDM A production. Table S8. The dose of important factors in response surface analysis. Table S9. Analysis of variance (ANOVA) for the second-order polynomial model. Figure S1. Phylogenetic tree of S. somaliensis SCSIO ZH66 based on 16S rRNA sequences. Figure S2. Phenotypes of strain 
ZH66-WT and strain ZH66-RIF1. Figure S3. HPLC traces of fermentation products of strain ZH66-RIF1 in different medium. Figure S4. UV spectrum of FDM $A$.

\section{Competing interests}

The authors declare that they have no competing interests.

\section{Authors' contributions}

$Y Z, H H$ and $X S$ performed the experiments. $Y Z$ wrote the draft manuscript. BW and $\mathrm{J} J$ sequenced the $16 \mathrm{~S}$ rDNA and the frd gene cluster. HT critically revised the manuscript. WL supervised the whole work and wrote the manuscript. All authors read and approved the final manuscript.

\section{Acknowledgments}

This work was supported by grants from the National High Technology Research and Development Program of China (2012AA092104), the National Natural Science Foundation of China (31171201), the NSFC-Shandong Joint Fund for Marine Science Research Centers (U1406402), and the State Key Laboratory of Microbial Resources Program, Institute of Microbiology, CAS (No. SKLMR-20110601)

\section{Author details}

'Key Laboratory of Marine Drugs, Ministry of Education of China, School of Medicine and Pharmacy, Ocean University of China, Qingdao 266003, China. ${ }^{2}$ CAS Key Laboratory of Marine Bio-resources Sustainable Utilization, Guangdong Key Laboratory of Marine Materia Medica, RNAM Center for Marine Microbiology, South China Sea Institute of Oceanology, Chinese Academy of Sciences, 164 West Xingang Road, Guangzhou 510301, China. ${ }^{3}$ State Key Laboratory of Microbial Resources, Institute of Microbiology, Chinese Academy of Sciences, Beijing 100101, China.

\section{Received: 16 February 2015 Accepted: 9 April 2015}

\section{Published online: 01 May 2015}

\section{References}

1. Jensen PR, Gontang E, Mafnas C, Mincer TJ, Fenical W. Culturable marine actinomycete diversity from tropical Pacific Ocean sediments. Environ Microbiol. 2005;7:1039-48.

2. Lam KS. New aspects of natural products in drug discovery. Trends Microbiol. 2007;15:279-89.

3. Fenical $W$, Jensen PR. Developing a new resource for drug discovery: marine actinomycete bacteria. Nat Chem Biol. 2006;2:666-73.

4. Hertweck C. Hidden biosynthetic treasures brought to light. Nat Chem Biol. 2009:5:450-2.

5. Hopwood DA. (2008) Small things considered: the tip of the iceberg. http://schaechter.asmblog.org/schaechter/2008/06/the-tip-of-the.html.

6. Ochi K, Hosaka T. New strategies for drug discovery: activation of silent or weakly expressed microbial gene clusters. Appl Microbiol Biot. 2013;97:87-98

7. Pan Y, Lu C, Dong H, Yu L, Liu G, Tan H. Disruption of rimP-SC, encoding a ribosome assembly cofactor, markedly enhances the production of several antibiotics in Streptomyces coelicolor. Microb Cell Fact. 2013;12:65.

8. Ochi K, Tanaka Y, Tojo S. Activating the expression of bacterial cryptic genes by rpoB mutations in RNA polymerase or by rare earth elements. J Ind Microbiol Biot. 2014;41:403-14

9. Hosaka T, Ohnishi-Kameyama M, Muramatsu H, Murakami K, Tsurumi Y, Kodani S, et al. Antibacterial discovery in actinomycetes strains with mutations in RNA polymerase or ribosomal protein S12. Nat Biotechnol. 2009;27:462-4

10. Fu P, Jamison M, La S, MacMillan JB. Inducamides A-C, Chlorinated alkaloids from an RNA polymerase mutant strain of Streptomyces sp. Org Lett. 2014;16:5656-9.

11. Pandey RC, Toussaint MW, Stroshane RM, Kalita C, Aszalos AA, Garretson AL, et al. Fredericamycin A, a new antitumor antibiotic. I. Production, isolation and physicochemical properties. J Antibiot. 1981;34:1389-401.

12. Misra R, Pandey RC, Hilton BD, Roller PP, Silverton JV. Structure of fredericamycin A, an antitumor antibiotic of a novel skeletal type; spectroscopic and mass spectral characterization. J Antibiot. 1987;40:786-802
13. Liou Y-C, Ryo A, Huang H-K, Lu P-J, Bronson R, Fujimori F, et al. Loss of Pin function in the mouse causes phenotypes resembling cyclin D1-null phenotypes. Proc Natl Acad Sci U S A. 2002;99:1335-40.

14. Latham MD, King CK, Gorycki P, Macdonald TL, Ross WE. Inhibition of topoisomerases by fredericamycin A. Cancer Chemoth Pharm. 1989;24:167-71.

15. Clive D, Angoh AG, Bennett SM. Radical spirocyclization: synthesis of an appropriately oxygenated spiro compound related to the antitumor antibiotic fredericamycin A. J Org Chem. 1987;52:1339-42.

16. Clive DL, Tao Y, Khodabocus A, Wu Y, Angoh AG, Bennett SM, et al. Total synthesis of crystalline ( \pm )-fredericamycin $A$. Use of radical spirocyclization. J Am Chem Soc. 1994;116:11275-86.

17. Boger DL, Hueter O, Mbiya K, Zhang M. Total synthesis of natural and ent-fredericamycin A. J Am Chem Soc. 1995;117:11839-49.

18. Wendt-Pienkowski E, Huang Y, Zhang J, Li B, Jiang H, Kwon H, et al. Cloning, sequencing, analysis, and heterologous expression of the fredericamycin biosynthetic gene cluster from Streptomyces griseus. J Am Chem Soc. 2005;127:16442-52.

19. Chen $Y$, Luo Y, Ju J, Wendt-Pienkowski E, Rajski SR, Shen B. Identification of fredericamycin E from Streptomyces griseus: Insights into fredericamycin A biosynthesis highlighting carbaspirocycle formation. J Nat Prod. 2008;71:431-7.

20. Chen Y, Wendt-Pienkowski E, Shen B. Identification and utility of FdmR1 as a Streptomyces antibiotic regulatory protein activator for fredericamycin production in Streptomyces griseus ATCC 49344 and heterologous hosts. J Bacteriol. 2008;190:5587-96.

21. Chen Y, Wendt-Pienkoski E, Rajski SR, Shen B. In vivo investigation of the roles of FdmM and FdmM1 in fredericamycin biosynthesis unveiling a new family of oxygenases. J Biol Chem. 2009;284:24735-43.

22. Hunter WG, Koehler TL. Response surface methodology. In: Juran JM, Gryna FM, editors. Quality control handbook. New York: McGraw-Hill Book Company; 1979. p. 1-12.

23. Wells ME. Response surface methodology and subjective data. In: Powers JJ, Moskowitz HR, editors. Correlating sensory objective measurements-new methods for answering old problems. Philadelphia: American Society for Testing of Materials; 1976. p. 73-80.

24. Bezerra MA, Santelli RE, Oliveira EP, Villar LS, Escaleira LA. Response surface methodology (RSM) as a tool for optimization in analytical chemistry. Talanta. 2008;76:965-77.

25. Kim OS, Cho YJ, Lee K, Yoon SH, Kim M, Na H, et al. Introducing EzTaxon-e: a prokaryotic $16 \mathrm{~S}$ rRNA gene sequence database with phylotypes that represent uncultured species. Int J Syst Evol Micr. 2012;62:716-21.

26. Tanaka Y, Kasahara K, Hirose Y, Murakami K, Kugimiya R, Ochi K. Activation and products of the cryptic secondary metabolite biosynthetic gene clusters by rifampin resistance $(r p o B)$ mutations in actinomycetes. J Bacteriol. 2013;195(13):2959-70.

27. Hosoya Y, Okamoto S, Muramatsu H, Ochi K. Acquisition of certain streptomycin-resistant (str) mutations enhances antibiotic production in bacteria. Antimicrob Agents Chemother. 1998;42(8):2041-7.

28. Zaleta-Rivera K, Charkoudian LK, Ridley CP, Khosla C. Cloning, sequencing, heterologous expression, and mechanistic analysis of A-74528 biosynthesis. J Am Chem Soc. 2010;132:9122-8.

29. Kieser T, Bibb MJ, Buttner MJ, Chater KF, Hopwood DA. Practical Streptomyces Genetics. Norwich: The John Innes Foundation; 2000.

30. Tamura K, Stecher G, Peterson D, Filipski A, Kumar S. MEGA6: molecular evolutionary genetics analysis version 6.0. Mol Biol Evol. 2013;30:2725-9.

31. Ishikawa J, Hotta K. FramePlot: a new implementation of the frame analysis for predicting protein-coding regions in bacterial DNA with a high $\mathrm{G}+\mathrm{C}$ content. FEMS Microbiol Lett. 1999;174:251-3.

32. Altschul SF, Gish W, Miller W, Myers EW, Lipman DJ. Basic local alignment search tool. J Mol Biol. 1990;215:403-10.

33. Tyssedal J. Plackett-Burman designs. In: Ruggeri F, Kenett RS, Faltin FW, editors. Encyclopedia of statistics in quality and reliability. New York: Wiley; 2008. p. 1361-5.

34. Kalil S, Maugeri F, Rodrigues M. Response surface analysis and simulation as a tool for bioprocess design and optimization. Process Biochem. 2000;35:539-50.

35. Gilmour SG. Response surface designs for experiments in bioprocessing. Biometrics. 2006;62:323-31. 